\title{
Optimization of Superconducting Focusing Quadrupoles for the High Current Experiment
}

\author{
GianLuca Sabbi, Steve Gourlay, Chen-yu Gung, Ray Hafalia, Alan Lietzke, Nicolai Martovetski, \\ Sara Mattafirri, Rainer Meinke, Joseph Minervini, Joel Schultz, and Peter Seidl
}

\begin{abstract}
The Heavy Ion Fusion (HIF) program is progressing through a series of physics and technology demonstrations leading to an inertial fusion power plant. The High Current Experiment (HCX) at Lawrence Berkeley National Laboratory is exploring the physics of intense beams with high line-charge density. Superconducting focusing quadrupoles have been developed for the HCX magnetic transport studies. A baseline design was selected following several pre-series models. Optimization of the baseline design led to the development of a first prototype that achieved a conductor-limited gradient of 132 $\mathrm{T} / \mathrm{m}$ in a $70 \mathrm{~mm}$ bore, without training, with measured field errors at the $0.1 \%$ level. Based on these results, the magnet geometry and fabrication procedures were adjusted to improve the field quality. These modifications were implemented in a second prototype. In this paper, the optimized design is presented and comparisons between the design harmonics and magnetic measurements performed on the new prototype are discussed.
\end{abstract}

Index Terms - Superconducting accelerator quadrupole, Heavy Ion Accelerator, Inertial Fusion Energy.

\section{INTRODUCTION}

$\mathrm{H}$ $\mathrm{CX}$ is designed to explore the physics of intense beams with driver-scale line-charge density $\left(0.2^{\circ} \mu \mathrm{C} / \mathrm{m}\right)$ and pulse duration $(\tau \geq 4 \mu s)$ [1]. The main objective of magnetic transport experiments in HCX is to investigate the effects due to electrons trapped in the potential well of the ion beam. A minimum field gradient of $84.2 \mathrm{~T} / \mathrm{m}$ over a magnetic length of $10.1 \mathrm{~cm}$ was specified for the superconducting quadrupoles [2]. The required coil aperture is $70 \mathrm{~mm}$. During the last several years, a collaboration of Lawrence Berkeley National Laboratory (LBNL), Lawrence Livermore National Laboratory (LLNL), MIT Plasma Science and Fusion Center and Advanced Magnet Lab (AML) has been developing magnets based on Niobium-Titanium (NbTi) conductor for HCX and future HIF applications. Following analysis and comparison of

Manuscript received September 20,2005. This work was supported by the Office of Energy Research, US DOE, at LBNL under contract number DEAC02-05CH1 1231, at LLNL under contract W-7405-Eng-48, and at MIT under contract number DE-FC02-93-ER54186.

G. Sabbi (phone: 510-495-2250; e-mail: GLSabbi@lbl.gov), S. Gourlay, R. Hafalia, A. Lietzke, S. Mattafirri, P. Seidl are with Lawrence Berkeley National Laboratory, Berkeley, CA.

N. Martovetsky is with Lawrence Livermore National Laboratory, Livermore, $\mathrm{CA}$.

C. Gung, J. Minervini, J. Schultz are with MIT Plasma Science and Fusion Center, Cambridge, MA.

R. Meinke is with Advanced Magnet Lab, Palm Bay, FL. several design concepts, prototype quadrupoles of two different types were fabricated and tested. A 2-layer racetrack design, developed by LLNL, was finally selected and further improved [3]. A prototype of the improved design (HCX-C) was fabricated by AML and tested at LBNL [4]. HCX-C reached its conductor-limited field of $7 \mathrm{~T}$ in the NbTi coil, corresponding to a gradient of $132 \mathrm{~T} / \mathrm{m}$. Following this test, the field quality was optimized by adjusting the magnet geometry and improving the fabrication procedures. These changes were implemented in a new prototype (HCX-D). In this paper, the HCX-D magnet design and test results will be presented and discussed.

\section{BASELINE DESIGN}

\section{A. General features}

The magnet design was developed taking into account both the specific objectives of the HCX experiment and the general requirements for application to future driver accelerators [5]. The coil is composed of eight double-layer racetrack windings (two for each quadrant) connected in series by soldered lap joints. Each sub-coil is wound around an iron core and housed in a mitered aluminum holder. The iron core is split in several sections, and wedges are inserted in between sections to preload the coil against the holder. The inner and outer windings of each quadrant are vacuum pressure impregnated with epoxy resin to form four monolithic sub-assemblies (coil modules). The mitered corners of the coil holders allow the four modules to be combined in a square assembly. A 4-piece iron yoke surrounds the coil and a welded stainless steel outer shell provides mechanical support.

\section{B. HCX-C prototype}

With respect to the pre-series models, HCX-C incorporated several design improvements. The coil ends were modified from continuous arcs to tight bends followed by straight segments, to increase the integrated gradient and improve the field quality. The coil holder material was changed from stainless steel to a less expensive, high strength aluminum alloy. The structural tube used in the bore of previous prototypes to provide internal support to the coils was removed. The superconducting strand was changed from SSCouter to SSC-inner type. The strand was drawn from $0.808 \mathrm{~mm}$ to $0.648 \mathrm{~mm}$, for match the cable parameters developed for pre-series models wound with SSC-outer strand. The cable is 
composed of 13 strands, has a nominal width of $4.05 \mathrm{~mm}$ and a thickness of $1.17 \mathrm{~mm}$. Turn-to-turn insulation is provided by a fiberglass sleeve with a nominal thickness of $0.12 \mathrm{~mm}$. The use of SSC-inner strand with low copper fraction allowed to achieve a maximum gradient of $132 \mathrm{~T} / \mathrm{m}$ in $\mathrm{HCX}-\mathrm{C}$, with an effective magnetic length of $105.4 \mathrm{~mm}$ for a coil physical length of $125 \mathrm{~mm}$.
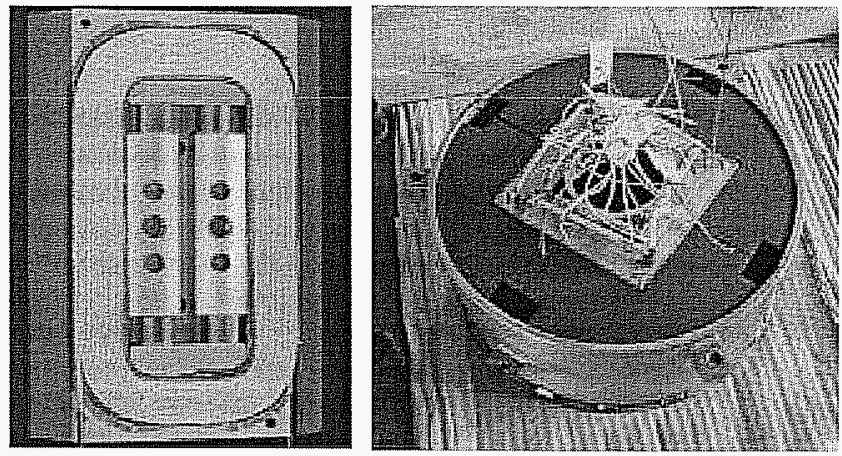

Fig. 1.Racetrack winding (left); final assembly of coil, yoke and shell (right).

\section{FIELD REPRESENTATION}

The HCX required field quality was specified in terms of axial integrals of the 3D magnetic field components [2]. For any longitudinal field integral calculated at $25 \mathrm{~mm}$ radius and $0<\theta<2 \pi$, a maximum deviation of $0.5 \%$ from the ideal quadrupole field at that location is allowed. The use of integrated field errors is well suited to short magnets with strong longitudinal field variations, and implicitly allows field error compensation between the magnet straight section and ends. Simulation studies of intense beams have shown that minimization of local field errors is desirable but not needed for the HCX application provided the integrated error is in the range specified.

For both design optimization and magnetic measurement purposes, the field is typically represented in terms of harmonic coefficients, defined by the power series expansion:

$$
\int\left(B_{y}+i B_{x}\right) d z=B_{2} 10^{-4} \sum_{n=1}^{\infty} \bar{c}_{n}\left(\frac{x+i y}{r_{0}}\right)^{n-1}
$$

where $B_{x}$ and $B_{y}$ are transverse field components, the integral extends over the entire magnetic length, $B_{2}$ is the quadrupole field, and $\bar{c}_{n}=b_{n}+i a_{n}$ are multipole coefficients, expressed in $10^{-4}$ "units" of the quadrupole component. Only the harmonic components $\mathrm{b}_{2 n+4}$ are allowed by the quadrupole symmetry. The other harmonics appear due to departures from perfect quadrupole symmetry, which may originate from either the magnet design or fabrication tolerances. The magnetic mid-planes of the quadrupole field lie along the $x$ and $y$-axes, and the $z$-axis is directed from the return end towards the lead end. Both measurements and calculations are longitudinally integrated over the length of the measurement coil. A reference radius $r_{0}$ of $22 \mathrm{~mm}$ was defined, corresponding to the radius of the available measurement probe.

\section{DESIGN OPTIMIZATION}

The HCX-C measurements for the allowed harmonics were in good agreement with calculations [4]. The main field errors at the $2.5 \mathrm{kA}$ reference current were generated by the 12 -pole $\left(b_{6}\right)$ and 20-pole $\left(b_{10}\right)$ components, for which corrections of 8.1 units and 8.7 units, respectively, were required. Two strategies were considered to generate these corrections, involving modifications of either the coil or the iron pole geometry. The coil design is constrained by the minimum thickness of the coil holder at its mitered corner (magnetic mid-plane) to prevent excessive bending and stress concentration. However, modifications of the iron pole geometry at selected locations can also contribute to generating the required corrections. After a detailed 3D analysis using the finite element code TOSCA, the following modifications were implemented (Fig. 2):

- three turns (for each layer) were eliminated from the inner coil, and one turn (for each layer) was eliminated from the outer coil. For both coils, the position of the mid-plane turns is unchanged: the turns are removed at the pole;

- two rectangular pockets were introduced in the iron pole of the inner coils, on the surface facing the bore. The pockets are $2.95 \mathrm{~mm}$ deep, $12 \mathrm{~mm}$ wide and $100 \mathrm{~mm}$ long.

In addition, the outer perimeters of the pole-islands were modified to fit the new profile of the coils, and the minimum bend radii for both coils were increased from $6 \mathrm{~mm}$ to $9 \mathrm{~mm}$ to facilitate winding.

Although the $b_{6}$ and $b_{10}$ components represented the main systematic contributions to the field errors in HCX-C, the optimization required close attention to the $b_{14}$ component. The HCX-C design had an integrated $b_{14}$ of -0.66 units at 22 $\mathrm{mm}$. Since $b_{14}$ rapidly increases with radius, it can become the dominant error for beams with high aperture filling factor. In fact, the position of the iron pocket which would be the most favorable to correct $b_{6}$ and $b_{10}$, is not accessible, since it would make $b_{14}$ significantly higher. Figure 3 shows a 2D calculation of the effect of a square cut (with $1 \mathrm{~mm}$ side) on the three main harmonics, as a function of the cut position. The requirement to limit the $b_{14}$ component constrains the shape and position of the iron cut-out, leading to more pronounced modifications of the coil geometry than were originally anticipated.

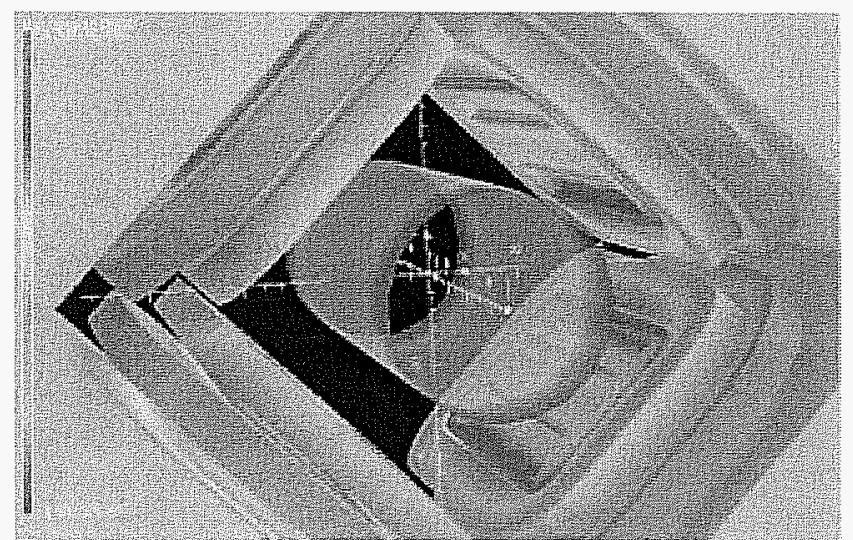

Fig. 2. FEM model showing the features used for field quality optimization. 


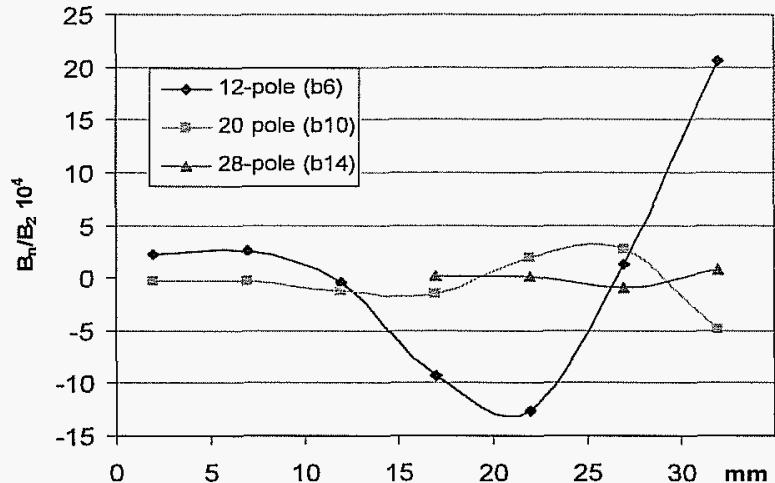

Fig. 3. Effect of a $1 \mathrm{~mm}$ square cut on the surface of the iron core on the allowed harmonics, as function of the cut position.

With the new design, the calculated $b_{6}$ and $b_{10}$ harmonics at a reference current of $2.5 \mathrm{kA}$ and $22 \mathrm{~mm}$ radius are reduced from 8-9 units to less than one unit. A small improvement of the $\mathrm{b}_{14}$ harmonic is also obtained. Saturation effects are comparable with the previous design: $b_{14}$ essentially does not depend on current; $b_{10}$ is in the range of -0.8 to 0.0 units between $2 \mathrm{kA}$ and $3 \mathrm{kA} ; \mathrm{b}_{6}$ is in the range of +5 to -5 units in the same current interval. This effect is mainly due to the saturation of the iron cores (inner and outer) and as such it is difficult to correct. However, it is possible to tune the $b_{6}$ to essentially zero at the operating current of choice with a small change of the depth of the iron pole cut-out. The other harmonics are not significantly affected by this change. Additional control of the non-allowed harmonics may be obtained by implementing a magnetic shim correction scheme similar to those developed for interaction region quadrupoles of high-energy colliders [6-7]. The cut-outs introduced in the inner pole-island for control of the systematic harmonics (Fig. 2 ans 3) are also suitable for housing the magnetic shims.

The transfer function (integrated gradient vs. current) decreases by about $9 \%$, due to the decrease in the number of turns, the increase of the minimum bending radius, and the cutout in the iron pole. However, the peak field (still located in the outer coil) also decreases by a similar amount. In addition, the peak field is better balanced between the inner and the outer coils (the difference in peak field is reduced from $9 \%$ to $5 \%)$. As a result, the quad focusing power does not decrease in a significant way $(-3 \%)$. The conductor volume is reduced by $12 \%$. The $50 \%$ increase of the minimum bending radius significantly facilitates coil winding.

\section{FABRICATION PROCEDURES}

The non-allowed harmonics observed in HCX-C were larger than expected based on Monte Carlo simulations, assuming conductor displacements uniformly distributed in the range of $\pm 100 \mu \mathrm{m}$ [4]. In order to better control the geometrical tolerances, and at the same time reduce the magnet cost, a new coil fabrication procedure was implemented by AML. With this method, the coils are wound around a monolithic poleisland, and vacuum impregnated in a precise mold to obtain an accurate and reproducible geometry. The impregnated coils are later inserted in aluminum holders, which are pre-heated to

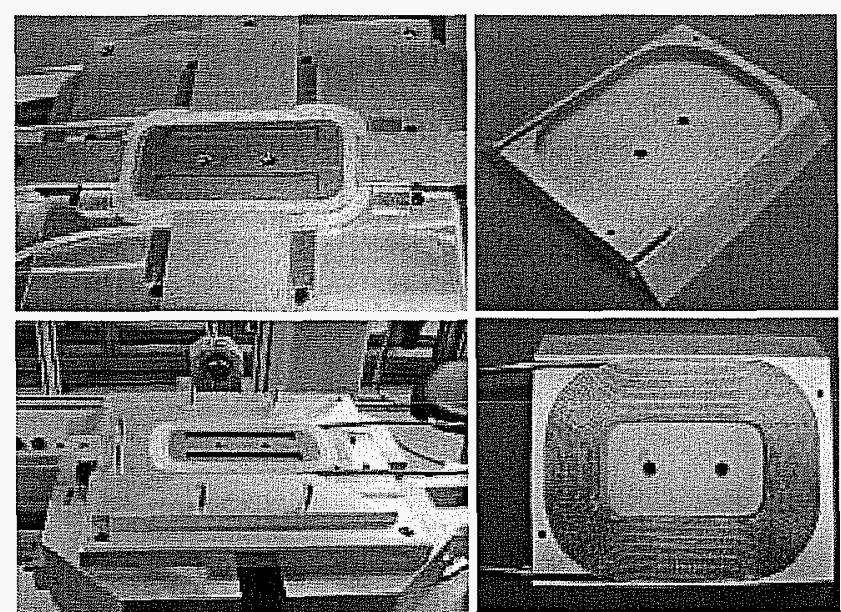

Fig. 4. HCX-D coil fabrication. Coil winding (top left); impregnation mold (bottom left); coil holder (top right); completed coil module (bottom right).

a temperature of $200^{\circ} \mathrm{C}$ to obtain sufficient clearance for coil insertion. At room temperature, there is a small interference between the coil and holder dimensions, resulting in a tight fit with no gaps. As for the previous prototype, the differential contraction coefficient between the iron pole and the aluminum coil holder provides additional coil pre-load after cool-down to $4.2 \mathrm{~K}$. The new procedure results in fewer parts, simpler fabrication steps and a more precise coil geometry. However, the pre-load, previously obtained at the assembly stage using a segmented pole-island with wedges (Fig. 1) is lost. Experimental validation of the magnet performance with the new coil fabrication method is therefore required.

\section{TEST REsULts}

\section{A. Quench performance}

The HCX-D performance was severely limited by quenches starting in one of the joints (connecting the inner and outer layer sub-coils of quadrant \#4) and propagating to the two adjacent coils. Voltage taps placed on both sides of this joint showed a voltage increase during the current ramps corresponding to very high resistance, about $62 \mathrm{n}$-Ohms. In fact, several other HCX-D joints also showed abnormally high resistance, in particular considering that all previous $\mathrm{HCX}$ prototypes were consistently below $1 \mathrm{n}-\mathrm{Ohm}$. At ramp rates of $5-20 \mathrm{~A} / \mathrm{s}$, the quench current was about $1.95 \mathrm{kA}$ or $63 \%$ of the calculated short sample limit $(3.1 \mathrm{kA})$. A maximum quench current of $67 \%$ of the short sample limit was recorded for very high ramp rates $(600 \mathrm{~A} / \mathrm{s})$ consistent with quenching due heat generation in the joint. Unfortunately, the HCX-D joints were enclosed in glass-filled epoxy for mechanical support, making a repair extremely difficult. The low quench currents prevented from testing the adequacy of the new fabrication method in providing mechanical support to the conductor against magnetic forces. However, magnetic measurements could be performed up to sufficiently high currents to provide a basic verification of the field quality for the new design. 


\section{B. MagneticMeasurements}

Magnetic measurements were performed using the LBNL vertical drive, rotating coil system. A $44.5 \mathrm{~mm}$ diameter, 82 $\mathrm{cm}$ long rotating probe, fabricated for the US-LHC quadrupole R\&D program [8], was provided by Fermilab. Details of the probe design and the measurement system are provided in [4]. The harmonic components were normalized to a reference radius of $22 \mathrm{~mm}$ to compare them with calculations.

The measured transfer function (Fig. 5) shows that despite severe limitations due to splice heating, HCX-D approached the minimum integrated gradient of $8.5 \mathrm{~T}$ which was specified for HCX. The allowed harmonics measured at $1.9 \mathrm{kA}$ showed a considerable improvement with respect to the previous prototype. In particular, the 20-pole $\left(b_{10}\right)$ component was reduced from 8.7 unit in HCX-C to 0.8 units in HCX-D. The measured 12-pole $\left(b_{6}\right)$ was 2.9 units. As it was already mentioned, saturation effects are expected to cause a monotonic decrease of the $b_{6}$ component in the current range of $2 \mathrm{kA}$ to $3 \mathrm{kA}$. Therefore, based on the $1.9 \mathrm{kA}$ measurement, a $b_{6}$ of about -2 units is expected at $2.5 \mathrm{kA}$, to be compared with 8 units in $\mathrm{HCX}-\mathrm{C}$. Although the integrated gradient achieved at $1.9 \mathrm{kA}$ is sufficient for $\mathrm{HCX}$ operation, a nominal design current of $2.5 \mathrm{kA}$ was chosen for the prototype magnets since there is a strong incentive to increase the focusing power in both HCX and future HIF applications.

Table I shows the measured non-allowed harmonics in HCX-D. These harmonics can be correlated to random field errors due to manufacturing tolerances. The table also shows the non allowed harmonics measured in $\mathrm{HCX}-\mathrm{C}$ and the results of Monte Carlo simulations to estimate these errors. In the first case, each conductor block (half-coil) in the magnet crosssection is randomly displaced with respect to its design position, assuming a flat distribution along each axis within a $\pm 100 \mu \mathrm{m}$ range. In the second case, each quadrant module (a sub-assembly composed of one inner and one outer coil) is displaced by the same amount. For each case, five hundred cross-sections were generated using ROXIE [9], and the average and rms values of their harmonics were calculated. The measured harmonics for $n=3, n=5, n=8$ and $n=9$ are considerably reduced with respect to $\mathrm{HCX}-\mathrm{C}$ and are consistent with the random error estimates. However, the $n=4$ and $n=7$ components are higher, and correspond to about 4 times the calculated sigma based on random displacements. Further analysis is required to understand the cause of these two harmonics.

TABLE I

NON-ALLOWED HARMONICS VS RANDOM ERRORS ( 1 SIGMA, $\left|c_{n}\right|$ units)

\begin{tabular}{ccccc}
$\begin{array}{c}\text { Order } \\
n\end{array}$ & $\begin{array}{c}\text { HCX-D } \\
\text { Measured }\end{array}$ & $\begin{array}{c}\text { HCX-C } \\
\text { Measured }\end{array}$ & $\begin{array}{c}\text { Random-Block } \\
\pm 100 \mu \mathrm{m}\end{array}$ & $\begin{array}{c}\text { Random-Quadr. } \\
\pm 100 \mu \mathrm{m}\end{array}$ \\
\hline 3 & 2.4 & 5.3 & 2.7 & 6.5 \\
4 & 6.6 & 2.5 & 1.8 & 1.8 \\
5 & 0.8 & 7.0 & 0.8 & 0.3 \\
7 & 1.4 & 0.6 & 0.2 & 0.5 \\
8 & 0.4 & 1.0 & 0.1 & 0.3 \\
9 & 0.4 & 2.8 & 0.05 & 0.1 \\
\hline
\end{tabular}

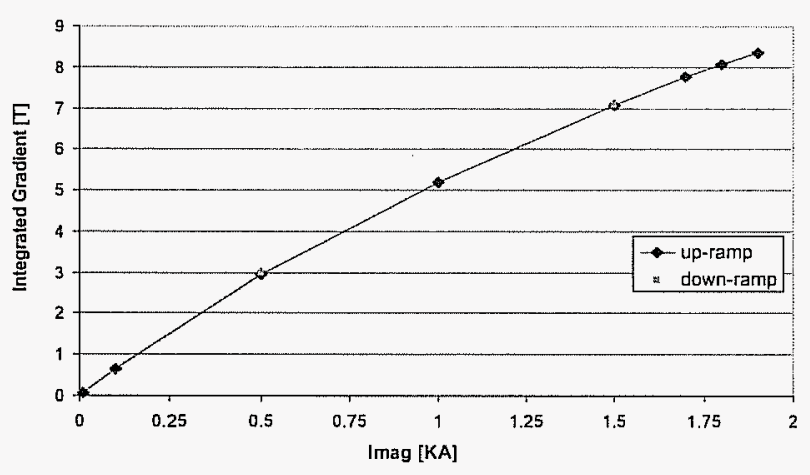

Fig. 5. HCX-D integrated gradient vs. current.

\section{CONCLUSIONS}

The design and test results of a prototype superconducting quadrupole for HIF applications were described. The main goal for this magnet was improving the field quality through design changes and new fabrication procedures. Magnetic measurements confirmed a strong reduction of the allowed harmonics, along with some improvement of the non-allowed components. Unfortunately, premature quenching due to heating generated in one of the inter-coil joints limited the magnet performance to well below its short sample limit. A new prototype is required to demonstrate acceptable quench performance with the new fabrication method.

\section{REFERENCES}

[1] P.A. Seidl; D. Baca; F.M. Bieniosek; C.M. Celata; A. Faltens; L.R. Prost; G. Sabbi; W.L. Waldron, "The High Current Transport Experiment for Heavy Ion Inertial Fusion," Particle Accelerator Conference PAC 03 (2003) HIFAN 1245, LBNL-53014.

[2] S. Lund. G. Sabbi, P. Seidl, "Characterization of Superconducting Quadrupoles for the High Current Experiment," HCX Magnet Note 01 0222-01, February 2001.

[3] G. Sabbi, A. Faltens, A. Lietzke, P. Seidl, S. Lund, B. Manahan, N. Martovetsky, C. Gung, J. Minervini, J. Schultz, L. Myatt, R. Meinke, "Progress in the Development of Superconducting Quadrupoles for Heavy Ion Fusion", Laser and Particle Beams (2002), 20, 617-620.

[4] N. Martovetsky, R. Manahan, R. Meinke, L. Chiesa, A. Lietzke, G. Sabbi, P. Seidl, "Development and Testing of the Improved Focusing Quadrupole for Heavy Ion Fusion Accelerators", Proceedings of the MT-18 Conference, Morioka, October 2003.

[5] R. Bangerter, J. Barnard, T. Brown, A. Faltens, C. Gung, E. Lee, A. Lietzke, S. Lund, B. Manahan, N. Martovetsky, R. Meinke, J. Minervini, L. Myatt, A. Radovinsky, G. Sabbi, J. Schultz, P. Seidl, S. $\mathrm{Yu}$, "Parameters and Requirements of Superconducting Focusing Quadrupoles for Heavy Ion Fusion", IEEE Trans. Appl. Supercond. Vol. 13, No. 2, June 2003, pp. 1530-1535.

[6] R. Gupta, et al., "Tuning Shims for High Field Quality in Superconducting Magnets", $14^{\text {th }}$ Conference on Magnet Technology, Tampere, Finland, June 1995.

[7] G. Sabbi, J. DiMarco, A. Nobrega, T. Ogitsu, P. Schlabach, J. Tompkins, S. Yadav, "Correction of High Gradient Quadrupole Harmonics with Magnetic Shims", IEEE Trans. Appl. Superconduct., vol. 10, no. 1, March 2000, pp. 123-126.

[8] P. Schlabach et al, "Field Quality in Fermilab-built Models of Quadrupole Magnets for the LHC Interaction Regions", IEEE Trans. Appl. Superconduct., vol. 11, no. 1, March 2001, pp. 1566.

[9] S. Russenschuck, A computer program for the design of superconducting magnets", Proc. ACES Symposium, Monterey, 1995 and CERN AT/95-39, September 1995. 